\title{
Comparison of rapid immunoassays for rupture of fetal membranes
}

Irogue Igbinosa', Ferney A. Moore III', Cheri Johnson² and Jon E. Block ${ }^{3^{*}}$

\begin{abstract}
Background: Rupture of membranes (ROM) before the onset of uterine contractions, particularly in pregnancies less than 37 weeks gestational age, is a common diagnostic problem in obstetrical practice. Timely detection of ROM is vital to support gestational age-specific interventions to optimize perinatal outcomes and minimize the risk of serious complications such as preterm delivery, fetal distress and maternal/fetal infections. Rapid bedside immunoassay tests designed to detect amniotic fluid proteins in cervicovaginal fluids have emerged as valuable clinical tools to provide timely ROM diagnosis.

Methods: In this prospective observational study, two commercially-available immunoassay tests (ROM Plus ${ }^{\oplus}$, AmniSure ${ }^{\oplus}$ ) were evaluated concurrently in 111 pregnant women who presented with the chief complaint of ROM. Immunoassay results were compared to clinical parameters for determining ROM via comprehensive, retrospective clinical chart review. Diagnostic performance characteristics were calculated including sensitivity, specificity, positive predictive value (PPV), negative predictive value (NPV) and accuracy.

Results: Overall, diagnostic performance characteristics were robust and similar between ROM Plus ${ }^{\oplus}$ and AmniSure ${ }^{\oplus}$, respectively: sensitivity (96.4 and 89.3\%), specificity (98.8 and 100\%), PPV (96.4 and 100\%), NPV (98.8\% and 96.5) and accuracy (98.2 and 97.3\%). For term patients ( $\geq 37$ weeks gestation), the sensitivities were 93.8 and $81.3 \%$ and specificities were 97.1 and $100 \%$ for ROM Plus ${ }^{\circledR}$ and AmniSure ${ }^{\oplus}$, respectively. For preterm patients ( $<37$ weeks gestation), both immunoassay tests provided exact concordance with clinical confirmation of ROM resulting in 100\% diagnostic accuracy.
\end{abstract}

Conclusions: Both rapid immunoassay tests provided similarly excellent diagnostic accuracy for the rapid detection of ROM with only two discrepant results for ROM Plus ${ }^{\circledast}$ and three discrepant results for AmniSure ${ }^{\circledast}$ compared to clinical confirmation. The findings from this study recommend these tests for pregnant women presenting with suspected ROM to guide correct clinical management decisions to improve obstetrical and neonatal outcomes.

Trial registration: ClinicalTrials.gov, NCT02208011 (1 August 2014).

Keywords: ROM plus ${ }^{\oplus}$, Amnisure ${ }^{\oplus}$, Premature rupture of membranes, Point of care immunoassay, Insulin-like growth factor binding protein (IGFBP-1), Alpha-fetoprotein (AFP), Placental alpha microglobulin-1 (PAMG-1)

* Correspondence: jb@drjonblock.com

${ }^{3} 2210$ Jackson Street, Suite 401, San Francisco, CA 94115, USA

Full list of author information is available at the end of the article 


\section{Background}

Rupture of membranes (ROM) has significant clinical implications particularly if it occurs prior to 37 weeks gestation where it is accountable for $20-40 \%$ of preterm deliveries [1-3]. In fact, preterm premature ROM (ROM prior to the onset of labor) complicates approximately $2-20 \%$ of all pregnancies in the US [4]. Accurate and timely diagnosis of ROM is crucial in guiding appropriate medical management decisions to optimize perinatal outcomes and ameliorate the risk of adverse events [5-8].

When ROM is suspected, traditional diagnostic methods include the use of a sterile speculum examination to visualize leakage of amniotic fluid from the external cervical os or pooling of amniotic fluid in the posterior vaginal fornix, coupled with a microscopic evaluation of a collected specimen for evidence of ferning/crystallization, commonly referred to as the fern test, and $\mathrm{pH}$ testing of the fluid with nitrazine test paper $[6,9,10]$. While this approach has remained the standard of care for decades, the results can be equivocal, especially in patients presenting for obstetrical care greater than an hour from the time of suspected ROM [11]. In addition, the sterile speculum exam may be subjective, and it has been shown to have false negatives and false positives which complicate the accurate detection of ROM [6, 12-15]. The "gold standard" to confirm ROM is to inject indigo carmine into the amniotic sac during amniocentesis and then assess whether any blue fluid is visibly leaking from the cervical os or pooling in the vaginal vault [6]. Leakage of the blue stained fluid into the vagina within $20-30$ min confirmed by staining of a tampon is regarded as the definitive diagnosis of membrane rupture. As amniocentesis is an invasive procedure with an associated $0.1-2 \%$ risk of miscarriage, less invasive methods for ROM determination are preferable for routine evaluation.

Several point of care immunoassay tests have been developed, validated and commercialized for the rapid and accurate diagnosis of ROM. These tests detect specific proteins, such as alpha fetoprotein (AFP), found in high concentrations in amniotic fluid but at extremely low background concentrations in cervico-vaginal secretions [16]. The first generation of these tests employed a monoclonal antibody approach focusing on insulin-like growth factor binding protein-1 (IGFBP-1, aka placental protein 12 [PP12]) and placental alpha microglobulin-1 (PAMG-1) [17-21]. More recently, a combined monoclonal/polyclonal antibody immunoassay has been developed to detect two different proteins found in amniotic fluid at high concentrations [22, 23]. This study was undertaken to evaluate the diagnostic accuracy for ROM detection of an original monoclonal antibody immunoassay test compared with a newer monoclonal/ polyclonal test.

\section{Methods}

This study compared the diagnostic performance characteristics between two commercially-available, immunochromatographic methods for the detection of ROM: 1) a monoclonal immunoassay that detects the PAMG-1 (AmniSure ${ }^{\circ}$, QIAGEN N.V., Netherlands), and 2) a monoclonal/polyclonal immunoassay that detects the presence of IGFBP-1 and AFP (ROM Plus ${ }^{\circ}$, Clinical Innovations, Salt Lake City, UT USA).

One hundred eleven (111) pregnant women, $\geq 15$ weeks gestation presenting with a complaint of ROM were enrolled in this study between August 2014 and June 2015. Pregnant women with known placenta previa and/or active vaginal bleeding were excluded. This research adhered to Good Clinical Practice (GCP) guidelines and followed the recommendations of the Helsinki Declaration. Each patient provided informed consent before any studyrelated procedures were performed. This trial was prospectively registered at ClinicalTrials.gov (NCT02208011).

At the time of clinical presentation, both immunoassay tests were performed simultaneously on each patient to determine presence or absence of ROM. The methodological and procedural details of both tests have been detailed previously $[18,23,24]$. In accordance with institutional regulations, both immunoassays were evaluated by authorized and trained personnel in the central hospital laboratory. Pregnant women with discrepant results between the two immunoassay tests underwent a sterile speculum exam (if not previously performed) and sonographic evaluation of amniotic fluid (AFI or $2 \times 2$ pocket). The patient was considered clinically positive for ROM if either 1) amniotic fluid was seen leaking from the cervical os, or 2) if at least two of the following three clinical signs were present: visual pooling of fluid in the posterior vaginal fornix, positive nitrazine test, and/or microscopic evidence of ferning. A thorough review of the medical records after delivery was undertaken utilizing specific major and minor criteria, prior to establishing the diagnosis of ROM.

The following major criteria were used in the clinician's review process as highly suggestive of ROM:

- $\mathrm{AFI}<7$ or no $2 \times 2$ pocket

- $<48 \mathrm{~h}$ from initial exam to delivery

- evidence of chorioamnionitis and/or endomyometritis

Clinical confirmation, the final diagnosis of ROM, required the presence of at least two major criteria.

Diagnostic performance characteristics with corresponding 95\% confidence intervals $(\mathrm{CI})$ were calculated for each test including sensitivity, specificity, positive predictive value (PPV), negative predictive value (NPV) and accuracy (i.e., overall agreement) $[25,26]$. Performance 
characteristics were computed for the entire patient population as well as separately for subgroups of term ( $\geq 37$ weeks gestation) and preterm patients ( $<37$ weeks gestation). Additionally, comparable performance characteristics for the sterile speculum exam were computed among the subgroup of pregnant women who underwent this procedure as part of their clinical assessment.

\section{Results}

Of the 111 pregnant women in this study, 51 (46\%) were term patients ( $\geq 37$ weeks gestation) and $60(54 \%)$ were preterm $(<37$ weeks gestation). For the entire patient population, comparing ROM Plus ${ }^{\circledR}$ results with clinical confirmation per retrospective chart review resulted in overall excellent diagnostic accuracy with a sensitivity of 96.4\% (95\% CI: $81.7 \%, 99.9 \%)$ and a specificity of $98.8 \%$ (95\% CI: 93.5\%, 100\%) (Table 1). The corresponding PPV and NPV for the ROM Plus ${ }^{\circ}$ were $96.4 \%$ (95\% CI: 81.7\%, 99.9\%) and 98.8\% (95\% CI: 93.5\%, 100\%), respectively. Only two measurements were discordant between ROM Plus ${ }^{\circ}$ and clinical confirmation resulting in an overall accuracy of $98.2 \%$ (109 of 111).

Comparing the AmniSure ${ }^{\circ}$ results with clinical confirmation for all patients led to slightly lower sensitivity than ROM Plus ${ }^{\circ}(89.3 \%$, 95\% CI: 71.8\%, 97.7\%) but exact specificity (100\%, 95\% CI: 95.7\%, 100\%) (Table 2). The corresponding PPV and NPV for the AmniSure were 100\% (95\% CI: 86.3\%, 100\%) and 96.5\% (95\% CI: 90.1\%, 99.3\%), respectively. Three measurements were discordant between AmniSure ${ }^{\circ}$ and clinical confirmation resulting in an overall accuracy of $97.3 \%$ (108 of 111).

The diagnostic performance results for the subgroup of term patients are provided in Tables 3 and 4. Compared to clinical confirmation, the sensitivities were 93.8\% (95\% CI: 69.8\%, 99.8\%) and 81.3\% (95\% CI: $54.4 \%, 96.0 \%)$ and the specificities were $97.1 \%$ (95\% CI: $85.1 \%, 99.9 \%$ ) and $100 \%$ (95\% CI: $90 \%, 100 \%)$ for ROM Plus $^{\circ}$ and AmniSure ${ }^{\circ}$, respectively. The PPVs were 93.8\% (95\% CI: 69.8\%, 99.8\%) and 100\% (95\% CI: 75.3\%, $100 \%$ ) and the NPVs were $97.1 \%$ (95\% CI: $85.1 \%, 99.9 \%$ ) and $92.1 \%$ (95\% CI: 78.6\%, 98.3\%) for ROM Plus ${ }^{\circ}$ and AmniSure ${ }^{\circ}$, respectively. The corresponding accuracy was $96.1 \%$ (49 of 51) for ROM Plus ${ }^{\circ}$ and $94.1 \%$ (48 of 51) for AmniSure ${ }^{\circ}$.

Table 1 Diagnostic performance characteristics in all patients: ROM plus ${ }^{\circledast}$ vs. clinical confirmation

\begin{tabular}{llll}
\hline & \multicolumn{2}{l}{ Clinical confirmation } & \\
\cline { 2 - 3 } ROM Plus & Positive & Negative & Total \\
\hline Positive & 27 & 1 & 28 \\
Negative & 1 & 82 & 83 \\
Total & 28 & 83 & 111 \\
\hline
\end{tabular}

Sensitivity $=96.4 \%$

Specificity $=98.8 \%$
Table 2 Diagnostic performance characteristics in all patients: Amnisure $^{\oplus}$ vs. clinical confirmation

\begin{tabular}{llll}
\hline & \multicolumn{2}{l}{ Clinical confirmation } & \\
\cline { 2 - 3 } AmniSure & Positive & Negative & Total \\
\hline Positive & 25 & 0 & 25 \\
Negative & 3 & 83 & 86 \\
Total & 28 & 83 & 111 \\
\hline Sensitivity $=89.3 \%$ & & & \\
Specificity $=100 \%$ & & &
\end{tabular}

For preterm patients, both immunoassay tests provided exact concordance with clinical confirmation of ROM (100\%, 60 of 60) (Tables 5 and 6).

There were 73 patients $(66 \%)$ who underwent a sterile speculum exam in this study as a part of their initial evaluation. Compared to clinical confirmation, the sensitivity and specificity of the speculum exam were $72.2 \%$ (95\% CI: 46.5\%, 90.3\%) and 96.4\% (95\% CI: 97.5\%, 99.6\%) with a PPV and NPV of $86.7 \%$ (95\% CI: 59.5\%, 98.3\%) and 91.4\% (95\% CI: 81.0\%, 97.1\%), respectively. There were 7 discordant measurements resulting in an accuracy of $90.4 \%$.

\section{Discussion}

Point of care diagnostic testing has revolutionized the medical management of patients with emergent conditions in the acute care setting [27, 28]. Rapid dissemination of results facilitates clinical decision making and improved patient outcomes in settings that often require expedient decisions such as labor and delivery. Traditional diagnostic approaches for ROM such as the sterile speculum exam to identify fluid leakage, nitrazine $\mathrm{pH}$ testing and miscroscopic fern analysis all lack consistently sufficient diagnostic accuracy $[6,9,10,12-14]$. More importantly, ROM detection becomes increasingly ambiguous when more than an hour has elapsed since membrane rupture, underscoring the urgent need for rapid, point of care testing that can be performed easily in various clinical settings [11].

This is the first report of a concurrent evaluation of these two commercially-available immunoassay tests for the diagnosis of ROM. Comparable diagnostic accuracy was achieved by both assays with wide and overlapping

Table 3 Diagnostic performance characteristics in patients $\geq$ 37 weeks gestation: ROM plus ${ }^{\circledast}$ vs. Clinical confirmation

\begin{tabular}{llll}
\hline & \multicolumn{2}{l}{ Clinical confirmation } & \\
\cline { 2 - 3 } ROM Plus & Positive & Negative & Total \\
\hline Positive & 15 & 1 & 16 \\
Negative & 1 & 34 & 35 \\
Total & 16 & 35 & 51 \\
\hline
\end{tabular}

Sensitivity $=93.8 \%$

Specificity $=97.1 \%$ 
Table 4 Diagnostic performance characteristics in patients $\geq$ 37 weeks gestation: AmniSure ${ }^{\circledast}$ vs. clinical confirmation

\begin{tabular}{llll}
\hline & \multicolumn{2}{l}{ Clinical confirmation } & \\
\cline { 2 - 3 } AmniSure & Positive & Negative & Total \\
\hline Positive & 13 & 0 & 13 \\
Negative & 3 & 35 & 38 \\
Total & 16 & 35 & 51
\end{tabular}

Sensitivity $=81.3 \%$

Specificity $=100 \%$

confidence intervals. The findings are similar to previously published results showing strong diagnostic accuracy (sensitivity 99\%, specificity 91\%) for the more newly introduced ROM Plus ${ }^{\oplus}$ assay [22]. Additionally, Rogers et al. [29] also reported similar diagnostic performance characteristics for the ROM Plus ${ }^{\oplus}$ with a sensitivity of $100 \%$ and specificity of $94.8 \%$ in 75 pregnant patients presenting with suspected ROM.

Both assays provided exact diagnostic accuracy in preterm patients with all measurements in complete agreement between tests and uniformly concordant with clinical confirmation. The risk of complications and worse outcomes increases significantly with lower gestational age making early ROM diagnosis, especially in very early preterm (i.e., $\leq 34$ weeks gestation) patients, imperative [30].

Achieving a high level of diagnostic accuracy is particularly important in cases of equivocal membrane rupture as nearly one-quarter of all pregnant women ultimately diagnosed with ROM do not present with overt clinical evidence of ruptured membranes on initial presentation [10]. We found notably lower sensitivity for the sterile speculum exam alone suggesting a diminished ability to correctly identify patients with ROM. Due to their procedural ease of use, these point of care tests can be performed rapidly at the patients' bedside and provide essential information in understanding the initial clinical scenario of pregnant women with suspected ROM. This diagnostic approach may be very useful in low resource settings where rapid, point of care testing has been shown to be more accurate and cost-effective than a sterile speculum exam [31].

Table 5 Diagnostic performance characteristics in patients < 37 weeks gestation: ROM plus ${ }^{\circledast}$ vs. clinical confirmation

\begin{tabular}{llll}
\hline & \multicolumn{2}{l}{ Clinical confirmation } & \\
\cline { 2 - 3 } ROM plus & Positive & Negative & Total \\
\hline Positive & 12 & 0 & 12 \\
Negative & 0 & 48 & 48 \\
Total & 12 & 48 & 60
\end{tabular}

Sensitivity $=100 \%$

Specificity $=100 \%$
Table 6 Diagnostic performance characteristics in patients < 37 weeks gestation: AmniSure ${ }^{\circledR}$ vs. clinical confirmation

\begin{tabular}{llll}
\hline & \multicolumn{2}{l}{ Clinical confirmation } & \\
\cline { 2 - 3 } AmniSure & Positive & Negative & Total \\
\hline Positive & 12 & 0 & 12 \\
Negative & 0 & 48 & 48 \\
Total & 12 & 48 & 60 \\
\hline Sensitivity $=100 \%$ & & & \\
Specificity $=100 \%$ & & &
\end{tabular}

\section{Conclusions}

Both rapid immunoassay tests evaluated in this study provided excellent diagnostic accuracy for the rapid detection of ROM with only two discrepant results for ROM Plus $^{\oplus}$ and three discrepant results for AmniSure compared to clinical confirmation. Test performance was particularly robust in preterm patients. The findings from this study support a recommendation for these tests in patients presenting with suspected ROM to guide clinical management decisions to improve obstetrical and neonatal outcomes. Future studies with larger patients sample sizes and cost comparison analyses to traditional methods of ROM detection may be helpful as institutions decide on implementation of point of care immunoassays.

\section{Abbreviations}

AFP: Alpha fetoprotein; AFI: Amniotic fluid index; Cl: Confidence interval; GCP: Good clinical practice; IGFBP-1: Insulin-like growth factor binding protein-1; NPV: Negative predictive value; PAMG-1: Placental alpha macroglobulin-1; PP12: Placental protein 12; PPV: Positive predictive value; ROM: Rupture of membranes; US: United States

\section{Acknowledgements}

We are grateful to the LSU OB/GYN residency program house officers, faculty and the staff of Woman's Hospital for their contributions to this study including Patrick Tassin, Karli Boggs, Regina McCuthceon and Ekwutosi Okoroh. We thank Terry Meredith for assistance with data presentation.

\section{Funding}

Manuscript development was funded by Clinical Innovations. No funds were received by the investigators. The funding body, Clinical Innovations, was involved in the development of the study protocol and was able to review it prior to initiating the trial. They were also kept apprised of patient enrollment status, but were not privy to any results until the study was completed.

\section{Availability of data and materials}

The datasets analyzed during the current study are available from the corresponding author on reasonable request.

\section{Authors' contributions}

II and FAM were involved in the study design. II, FAM and CJ developed and approved the study protocol. II and CJ coordinated the study. JEB evaluated the study data. All authors participated in the development of this manuscript. All authors read and approved the final manuscript.

\section{Competing interests}

This study was funded by the producers of the ROM Plus ${ }^{\circledast}$ test (Clinical Innovations, Salt Lake City, UT USA). JEB is an independent advisor to Clinical Innovations and received financial support for the development of this manuscript. 


\section{Consent for publication}

Not applicable.

\section{Ethics approval and consent to participate}

This study was conducted in accordance with the Declaration of Helsinki. Institutional Review Board (IRB) approval for this study was obtained from Woman's Hospital and Louisiana State University School of Medicine (New Orleans, LA). All patients provided written informed consent prior to participation.

\section{Publisher's Note}

Springer Nature remains neutral with regard to jurisdictional claims in published maps and institutional affiliations.

\section{Author details}

'Department of Obstetrics \& Gynecology, Woman's Hospital, Louisiana State University Health Sciences, 500 Rue de la vie, Suite 414, Baton Rouge, LA 70817, USA. 'Woman's Hospital, 100 Woman's Way, Baton Rouge, LA 70817, USA. ${ }^{3} 2210$ Jackson Street, Suite 401, San Francisco, CA 94115, USA.

\section{Received: 13 October 2016 Accepted: 19 April 2017}

\section{Published online: 26 April 2017}

\section{References}

1. Bulletins-Obstetrics ACoP. ACOG Practice Bulletin No. 80: premature rupture of membranes. Clinical management guidelines for obstetrician-gynecologists. Obstet Gynecol. 2007;109(4):1007-19.

2. Mercer BM, Goldenberg RL, Meis PJ, Moawad AH, Shellhaas C, Das A, Menard MK, Caritis SN, Thurnau GR, Dombrowski MP, et al. The preterm prediction study: prediction of preterm premature rupture of membranes through clinical findings and ancillary testing. The national institute of child health and human development maternal-fetal medicine units network. Am J Obstet Gynecol. 2000;183(3):738-45.

3. Naeye RL, Peters EC. Causes and consequences of premature rupture of fetal membranes. Lancet. 1980;1(8161):192-4.

4. Waters TP, Mercer B. Preterm PROM: prediction, prevention, principles. Clin Obstet Gynecol. 2011;54(2):307-12.

5. Ananth CV, Oyelese Y, Srinivas N, Yeo L, Vintzileos AM. Preterm premature rupture of membranes, intrauterine infection, and oligohydramnios: risk factors for placental abruption. Obstet Gynecol. 2004;104(1):71-7.

6. Caughey AB, Robinson JN, Norwitz ER. Contemporary diagnosis and management of preterm premature rupture of membranes. Rev Obstet Gynecol. 2008;1(1):11-22.

7. Di Renzo GC, Roura LC, Facchinetti F, Antsaklis A, Breborowicz G, Gratacos E, Husslein P, Lamont R, Mikhailov A, Montenegro N, et al. Guidelines for the management of spontaneous preterm labor: identification of spontaneous preterm labor, diagnosis of preterm premature rupture of membranes, and preventive tools for preterm birth. J Matern Fetal Neonatal Med. 2011;24(5):659-67.

8. Garite TJ. Management of premature rupture of membranes. Clin Perinatol. 2001;28(4):837-47.

9. de Haan HH, Offermans PM, Smits F, Schouten HJ, Peeters LL. Value of the fern test to confirm or reject the diagnosis of ruptured membranes is modest in nonlaboring women presenting with nonspecific vaginal fluid loss. Am J Perinatol. 1994;11(1):46-50.

10. El-Messidi A, Cameron A. Diagnosis of premature rupture of membranes: inspiration from the past and insights for the future. J Obstet Gynaecol Can. 2010;32(6):561-9.

11. Friedman ML, McElin TW. Diagnosis of ruptured fetal membranes. Clinical study and review of the literature. Am J Obstet Gynecol. 1969;104(4):544-50.

12. Gorodeski IG, Haimovitz L, Bahari CM. Reevaluation of the $\mathrm{pH}$, ferning and Nile blue sulphate staining methods in pregnant women with premature rupture of the fetal membranes. J Perinat Med. 1982;10(6):286-92.

13. Reece EA, Chervenak FA, Moya FR, Hobbins JC. Amniotic fluid arborization: effect of blood, meconium, and pH alterations. Obstet Gynecol. 1984;64(2):248-50.

14. Rosemond RL, Lombardi SJ, Boehm FH. Ferning of amniotic fluid contaminated with blood. Obstet Gynecol. 1990;75(3 Pt 1):338-40.

15. Smith RP. A technic for the detection of rupture of the membranes. A review and preliminary report. Obstet Gynecol. 1976;48(2):172-6.

16. Mariona FG, Cabero L. Are we ready for a new look at the diagnosis of premature rupture of membranes? J Matern Fetal Neonatal Med. 2012;25(4):403-7.
17. Doret M, Cartier R, Miribel J, Massardier J, Massoud M, Bordes A, Moret S, Gaucherand P. Premature preterm rupture of the membrane diagnosis in early pregnancy: PAMG-1 and IGFBP-1 detection in amniotic fluid with biochemical tests. Clin Biochem. 2013:46(18):1816-9.

18. Lee SE, Park JS, Norwitz ER, Kim KW, Park HS, Jun JK. Measurement of placental alpha-microglobulin-1 in cervicovaginal discharge to diagnose rupture of membranes. Obstet Gynecol. 2007;109(3):634-40.

19. Palacio M, Kuhnert M, Berger R, Larios CL, Marcellin L. Meta-analysis of studies on biochemical marker tests for the diagnosis of premature rupture of membranes: comparison of performance indexes. BMC Pregnancy Childbirth. 2014;14:183.

20. Rutanen EM, Bohn H, Seppala M. Radioimmunoassay of placental protein 12: levels in amniotic fluid, cord blood, and serum of healthy adults, pregnant women, and patients with trophoblastic disease. Am J Obstet Gynecol. 1982;144(4):460-3.

21. Rutanen EM, Pekonen F, Karkkainen T. Measurement of insulin-like growth factor binding protein -1 in cervical/vaginal secretions: comparison with the ROM-check membrane immunoassay in the diagnosis of ruptured fetal membranes. Clin Chim Acta. 1993;214(1):73-81.

22. Thomasino $T$, Levi C, Draper M, Neubert AG. Diagnosing rupture of membranes using combination monoclonal/polyclonal immunologic protein detection. J Reprod Med. 2013;58(5-6):187-94.

23. McQuivey RW, Block JE. ROM plus((R)): accurate point-of-care detection of ruptured fetal membranes. Med Devices (Auckl). 2016;9:69-74.

24. Cousins LM, Smok DP, Lovett SM, Poeltler DM. AmniSure placental alpha microglobulin -1 rapid immunoassay versus standard diagnostic methods for detection of rupture of membranes. Am J Perinatol. 2005;22(6):317-20.

25. Altman DG, Bland JM. Diagnostic tests. 1: Sensitivity and specificity. BMJ. 1994;308(6943):1552.

26. Bossuyt PM, Reitsma JB, Bruns DE, Gatsonis CA, Glasziou PP, Irwig LM, Moher D, Rennie D, de Vet HC, Lijmer JG, et al. The STARD statement for reporting studies of diagnostic accuracy: explanation and elaboration. Ann Intern Med. 2003:138(1):W1-12.

27. Jahn UR, Van Aken H. Near-patient testing-point-of-care or point of costs and convenience? Br J Anaesth. 2003:90(4):425-7.

28. Price CP. Point of care testing. BMJ. 2001;322(7297):1285-8.

29. Rogers LC, Scott L, Block JE. Accurate point-of-care detection of ruptured fetal membranes: improved diagnostic performance characteristics with a monoclonal/polyclonal immunoassay. Clin Med Insights Reprod Health. 2016;10:15-8.

30. Practice bulletins No. 139: premature rupture of membranes. Obstet Gynecol. 2013;122(4):918-30.

31. Eleje GU, Ezugwu EC, Ogunyemi D, Eleje LI, Ikechebelu Jl, Igwegbe AO, Okonkwo JE, Ikpeze OC, Udigwe GO, Onah HE, et al. Accuracy and cost-analysis of placental alpha-microglobulin - 1 test in the diagnosis of premature rupture of fetal membranes in resource-limited community settings. J Obstet Gynaecol Res. 2015;41(1):29-38.

\section{Submit your next manuscript to BioMed Central and we will help you at every step:}

- We accept pre-submission inquiries

- Our selector tool helps you to find the most relevant journal

- We provide round the clock customer support

- Convenient online submission

- Thorough peer review

- Inclusion in PubMed and all major indexing services

- Maximum visibility for your research

Submit your manuscript at www.biomedcentral.com/submit
) Biomed Central 